\title{
O PESSEGUEIRO EM POMAR COMPACTO. IX. DEZ ANOS DE PRODUÇÃO DE CULTIVARES SOB PODA DRÁSTICA BIENAL ${ }^{1}$
}

\author{
WILSON BARBOSA ${ }^{2}$, FERNANDO ANTONIO CAMPO DALL'ORTO ${ }^{3}$, MÁRIO OJIMA4, RUI RIBEIRO DOS SANTOS ${ }^{5}$, \\ GEOVANITA PAULINO DA COSTA KALIL ${ }^{6}$, JOEL IRINEU FAHL E MARIA LUIZA CARVALHO CARELLI ${ }^{7}$
}

\begin{abstract}
RESUMO - O objetivo deste trabalho foi comparar o desenvolvimento vegetativo e reprodutivo de quatorze cultivares e seleções de pessegueiro (Prunus persica L. Batsch) e nectarineira cultivadas no espaçamento de $4 \times 1,5 \mathrm{~m}(1.667 \mathrm{pl} / \mathrm{ha})$, sob poda drástica bienal da copa. Pelos resultados obtidos no decênio 1986-95, verificou-se que as cultivares e seleções mais produtivas foram (número e kg de frutos/planta respectivamente): Aurora-1 (112; 9,2), IAC 680-178 (94; 8,5), IAC 680-13 (95; 8,1), Talismã $(99 ; 8,1)$, Régis $(98 ; 7,8)$ e Flordaprince $(91 ; 7,7)$. No ano agrícola de 1995 , as variáveis analisadas, área da secção transversal do tronco, área da folha, número e massa dos frutos por $\mathrm{cm}^{2} \mathrm{de}$ tronco, apresentaram diferenciação estatística entre cultivares e seleções. A poda drástica bienal da copa ocasionou, tanto em material precoce quanto mediano, diminuição de produção a cada duas safras, não chegando, porém, a prejudicar a produtividade média do pomar.
\end{abstract}

Termos para indexação: Prunus persica, prática cultural, seleções IAC, longevidade da planta, produtividade, vigor, área foliar.

\author{
THE PEACH MEADOW ORCHARD SYSTEM. \\ IX. YIELD OF CULTIVARS DURING TEN YEARS ON BIENNIAL DRASTIC PRUNING
}

\begin{abstract}
Fourteen peach (Prunus persica L. Batsch) and nectarine cultivars and selections, budded on Okinawa rootstock, were grown under the meadow orchard system, with biennial drastic pruning at $4 \times 1.5 \mathrm{~m}$ spacing. Results obtained during ten years of experiments (1986-95) clearly indicate that 'Aurora-1', IAC 680-178, IAC 680-13, 'Talismã', 'Régis' and 'Flordaprince' peaches were the best, with an average yield (fruits) per tree of $112,9.2 ; 94,8.5 ; 95,8.1 ; 99,8.1 ; 98,7.8$ and 91, 7.7 number and $\mathrm{kg} /$ tree respectively. The biennial drastic pruning decreased the yield of trees every two years, without affecting the average decennial productivity. In 1995, data on trunk cross-sectional area, fruits and mass per $\mathrm{cm}^{2}$ of trunk and leaf area presented significant differences among cultivars and selections.
\end{abstract}

Index terms: Prunus persica, cultural practice, IAC selections, longevity of tree, productivity, vigor, leaf area.

\footnotetext{
${ }^{1}$ Aceito para publicação em 22 de junho de 1998.

Pesquisa integrante do projeto IAC: "Pessegueiro: melhoramento genético e cultural".

${ }^{2}$ Biólogo, M.Sc., Seção de Fruticultura de Clima Temperado (SFCT), Instituto Agronômico (IAC), Caixa Postal 28, CEP 13001-970 Campinas, SP. Bolsista do CNPq. E-mail: wbarbosa@barao.iac.br ou barbosa@supernet.com.br

${ }^{3}$ Eng. Agr., M.Sc., SFCT, IAC. Bolsista do CNPq.

${ }^{4}$ Eng. Agr., Dr., SFCT, IAC. Bolsista do CNPq.

${ }^{5}$ Eng. Agr., Estação Experimental de Monte Alegre do Sul, IAC, Caixa Postal 50, CEP 13910-000 Monte Alegre do Sul, SP.

${ }^{6}$ Eng. Agr., M.Sc., estagiária, SFCT, IAC. Bolsista do CNPq.

${ }^{7}$ Eng. Agr., Dr., Seção de Fisiologia, IAC. Bolsista do CNPq.
}

\section{INTRODUÇÃO}

A técnica do cultivo de pessegueiros e nectarineiras em alta densidade de plantio vem sendo intensamente pesquisada e aperfeiçoada desde o início dos anos setenta (Erez, 1978; Bargioni et al., 1979). Nas últimas duas décadas, pesquisou-se o comportamento dessas fruteiras, em densidades populacionais de até cerca de 20.000 plantas por hectare, com diversos tipos de podas (Guerriero et al., 1980; Campo Dall'Orto et al., 1984; Barbosa et al., 1990b). O interesse científico mundial por tal modalidade cultural se justifica plenamente, tanto pela 
maior produtividade, logo nas primeiras frutificações, quanto pelas facilidades nos tratos culturais, que reduzem a mão-de-obra especializada no pomar.

Em cerca de cinqüenta trabalhos científicos e técnicos publicados sobre o assunto, foram relatados muitos resultados relevantes, sendo cultivares, tipos e épocas de poda os mais discutidos (Erez, 1985; Barbosa et al., 1989, 1991a, 1992, 1994, 1996; Loreti et al., 1991; Campo Dall'Orto et al., 1992). Ressalte-se que a poda drástica de copa não se constitui em regra geral na condução de pomar compacto. Nos países de clima temperado, os pessegueiros se desenvolvem com menor intensidade, e às vezes a poda drástica pode ser dispensada. Nas regiões de clima subtropical-tropical, ao contrário, as plantas se desenvolvem vigorosamente, formando imensas copas, em uma longa estação de crescimento. O conseqüente auto-sombreamento excessivo do dossel da copa enfraquece os ramos produtivos e compromete seriamente a produção da planta.

Além disso, a constituição extremamente vigorosa dos pessegueiros proporciona, em época precoce, acentuada competição espacial intercopas; assim, a prática da poda drástica constitui-se em fator indispensável ao sucesso do sistema. Há cerca de vinte anos, a Seção de Fruticultura de Clima Temperado, do IAC, vem pesquisando as melhores cultivares visando ao cultivo compensatório do pessegueiro no sistema de pomar compacto.

No tocante à adoção da poda drástica anual da copa, várias pesquisas demonstraram ser indispensável o emprego de cultivares de maturação precoce, com ciclo florada-colheita dos frutos abaixo de 100 dias. Sabe-se que, efetuando-se a colheita o mais cedo possível e sucedendo-a com podas das copas, há condições ecofisiológicas para brotação de novos ramos, diferenciação floral, floração e maturação dos frutos, respectivamente, em 1, 5, 9 e 12 meses de desenvolvimento (Barbosa et al., 1990c). Com esse comportamento, as cultivares de maturação mais precoce como Tropical e Flordaprince repetem, de forma normal, o ciclo reprodutivo nos anos subseqüentes (Barbosa et al., 1991b). Já em se utilizando cultivares de ciclo de maturação mediano como Aurora-2, constata-se deficiência no desenvolvimento da copa, diminuindo acentuadamente a frutificação da planta ao longo dos anos. Mesmo submetidas a uma poda anual mais antecipada, as cultivares do tipo maturação mediana ainda apresentam ciclo podacolheita dos frutos acima de doze meses, prejudicando a fase vegetativa seguinte (Barbosa, 1989). Foi, então, formulada a hipótese de que ao cultivo de pessegueiros de maturação mediana ou tardia se justificaria a tentativa de modificar a periodicidade da poda drástica da copa.

O objetivo deste trabalho foi comparar o desenvolvimento vegetativo e reprodutivo de quatorze cultivares e seleções de pessegueiro e nectarineira, em pomar compacto, com poda drástica bienal pós-colheita.

\section{MATERIAL E MÉTODOS}

Quatorze cultivares e seleções de pêssego e nectarina foram pesquisadas no sistema de pomar compacto, com poda drástica bienal, na Estação Experimental de Monte Alegre do Sul, do IAC, em latitude: $22^{\circ} 41^{\prime} \mathrm{S}$; longitude: $46^{\circ} 43^{\prime} \mathrm{W}$; e 40 horas anuais de frio abaixo de $7^{\circ} \mathrm{C}$ (Pedro Junior et al., 1979). As cultivares e seleções foram: pêssego - Aurora-1, Régis, Talismã, Flordaprince, Jóia-1, Tropical, Delicioso Precoce, IAC 680-178, IAC 680-13 e IAC 2380-55; nectarinas - Centenária, Rubro-sol (Sunred), Josefina e IAC N 2680-91. Dessa relação, quatro cultivares encontravam-se em exploração comercial no Estado de São Paulo, por ocasião da instalação do experimento, em 1985: Talismã e Rubro-sol, as mais tradicionais, que foram incluídas como referência, e Flordaprince e Jóia-1, então recém-apresentadas aos produtores. As demais seleções IAC achavam-se em fase de avaliações finais, sendo o lançamento como novas cultivares efetuado nos anos subseqüentes pela Seção de Fruticultura de Clima Temperado, do IAC (Ojima et al., 1988, 1993; Campo Dall'Orto et al., 1989; Barbosa et al., 1990a).

O lote experimental foi conduzido no espaçamento de $4 \times 1,5 \mathrm{~m}$ (1.667 plantas/ha), com cinco plantas por cultivar e seleção, as quais foram formadas em recipientes de plástico, tendo o pessegueiro Okinawa como porta-enxerto. Os pessegueiros e nectarineiras, conduzidos com cinco pernadas, foram podados a cada dois anos, a $50 \mathrm{~cm}$ do solo. A primeira poda drástica ocorreu em 1987; a partir daí, foi sempre realizada em anos ímpares, desde fins de outubro até meados de novembro, dependendo da cultivar. Todas as cultivares e seleções receberam tratos culturais rotineiros, como: pincelamento do tronco decepado, desbrota, capina, pulverização fitossanitária, irrigação no período reprodutivo, correção do solo, adubação e quebra de endodormência. Para facilitar o manejo, as plantas fo- 
ram rebaixadas, em invernos de anos pares, a cerca de $2,5 \mathrm{~m}$ de altura. No raleio, foram deixados dois e três frutos por ramo médio e vigoroso, respectivamente. Durante dez anos, de 1986 a 1995, efetuou-se, por safra, o controle da produção, em número e massa dos frutos, colhidos em cinco plantas por cultivar e seleção.

Em fins de outubro de 1995, décimo e último ano da pesquisa, foram controladas a área de secção transversal do tronco, o volume da copa, a área da folha, os frutos e a massa por $\mathrm{cm}^{2}$ de tronco. A área da secção transversal do tronco $\left(\mathrm{cm}^{2}\right)$ foi obtida convertendo-se a medida do diâmetro do tronco $(\mathrm{cm})$, determinada com um paquímetro a cerca de $20 \mathrm{~cm}$ do solo, com a tabela apresentada por Westwood (1978). O volume da copa foi calculado mediante as medidas de altura e largura, empregando-se a fórmula: $4 / 3 a b^{2}$, onde $a$ refere-se a $1 / 2$ do eixo maior, e $b, a^{1 / 2}$ do eixo menor (Westwood, 1978). A área da folha $\left(\mathrm{cm}^{2}\right)$ foi estimada pelo método da fotocópia, sendo a mesma conhecida por meio da interpolação das massas de um papel de $100 \mathrm{~cm}^{2}$ e da impressão recortada da folha (Reis \& Müller, 1979). A produção $/ \mathrm{cm}^{2}$ de tronco foi estimada dividindo-se o número e a massa média dos frutos pela área de secção transversal de tronco de cada planta. Efetuou-se a análise da variância, e as médias foram comparadas pelo teste de Tukey (5\%). Calculou-se, também, o coeficiente de correlação simples, nas principais características fisiológicas de produção.

\section{RESULTADOS E DISCUSSÃO}

Todos os pessegueiros e nectarineiras iniciaram a frutificação no segundo ano de desenvolvimento. Os pessegueiros Aurora-1 e IAC 680-178 apresentaram alta produtividade e potencial para cultivo em elevada densidade de plantio. Verificou-se, porém, em 1988, ano subseqüente à primeira poda drástica da copa, acentuada queda de produção, especialmente na seleção IAC 680-178. Isso, no entanto, não comprometeu seu desempenho produtivo no ciclo de 1989, pois com excelente regeneração da copa, esse material voltou a ter produção recorde (Tabelas 1 e 2). Essa queda de produção, ocorrida após as podas bienais, permaneceu evidente durante toda a experimentação, tornando-se regra para a maioria das cultivares.

Coincidentemente, as cinco cultivares e seleções mais produtivas, nos dez anos de experimentação, foram as do tipo polpa firme e de similar linhagem genealógica (Barbosa et al., 1997). Esses pesseguei- ros híbridos IAC apresentam em comum a descendência em 1aㅗ $2^{\underline{a}}$ ou $3^{\mathrm{a}}$ geração do Rei da Conserva, cultivar antiga, tipo conserva e extremamente rústica. São eles: Aurora-1, IAC 680-178, IAC 680-13, Régis e Talismã, com produções médias anuais, no decênio, em número e kg/planta, de 112 e 9,2; 94 e 8,$5 ; 95$ e 8,$1 ; 98$ e 7,8 e 99 e 8,1 , respectivamente. A queda de produção verificada nos anos pares, nesse grupo de pessegueiros, não comprometeu a produtividade média do pomar. Isto corresponde à produtividade decenal acumulada desde $130 \mathrm{t} / \mathrm{ha}$ no tocante à Régis, até 153 t/ha no tocante à Aurora-1, situando-se a de IAC 680-178, IAC 680-13 e Talismã entre esses limites. Essas cinco cultivares e seleções possuem ciclos florada e maturação dos frutos entre $95 \mathrm{e}$ 143 dias, sendo enquadradas nos grupos III (precoces) e IV (medianas), segundo a classificação atual (Barbosa et al., 1990d).

A cultivar Flordaprince, de maturação bem precoce, introduzida da Universidade da Flórida pelo Instituto Agronômico, também apresentou excelente comportamento, com produção média decenal de 91 frutos e $7,7 \mathrm{~kg} /$ planta. Essa cultivar, por sinal, vem apresentando resultados dos mais satisfatórios, em todos os plantios adensados experimentados (Campo Dall'Orto et al., 1984; Barbosa et al., 1996).

As cultivares Jóia-1, Centenária, Tropical e Delicioso Precoce apresentaram produções médias entre $6,2 \mathrm{~kg}$ e $6,7 \mathrm{~kg} /$ planta/ano. Estes valores correspondem a uma produtividade na faixa de $10 \mathrm{t} / \mathrm{ha}$, considerada apenas razoável para pomar compacto. As produções médias de algumas destas cultivares, podem, talvez, ser melhoradas com podas mais antecipadas. No presente experimento, optou-se em retardar um pouco a poda das cultivares "bem precoces" e "precoces", evitando-se a defasagem de crescimento em relação ao mediano. Com efeito, as brotações dos troncos dos diversos pessegueiros e nectarineiras, desenvolveram-se sadias e uniformes; mas ao serem podadas as copas entre fins de outubro e meados de novembro, as cultivares bem precoces tiveram produtividade um tanto prejudicada. Esse fato foi verificado também anteriormente quanto à cultivar Tropical, quando se retardou a poda drástica para fins de outubro, ocorrendo, então, uma queda de produção de $70 \%$ em relação às plantas podadas em fins de setembro (Barbosa et al., 1991b). 
Quanto à produtividade em pomares compactos, Bargioni et al. (1985) relatam que, em espaçamentos de 4 x $1 \mathrm{~m}, 4$ x 1,5 m e 4 × 2,0 m, um rendimento acima de 15 t/ha já seria satisfatório, o que é compatível com os resultados ora obtidos nas cultivares e sele- ções mais produtivas. Tanto esses autores como Recupero et al. (1985) concluíram que o espaçamento de $4 \times 1 \mathrm{~m}$ pode ser dos mais interessantes para se conciliar alta produtividade com melhor qualidade de frutos, além das facilidades nos tratos culturais.

TABELA 1. Número médio de frutos, por planta, de cultivares e seleções de pessegueiros e nectarineiras no espaçamento 4 x 1,5 m (1.667 pl/ha), durante o período de 1986 a 1995 . Estação Experimental de Monte Alegre do Sul, IAC ${ }^{1}$.

\begin{tabular}{lccccccccccc}
\hline Material & 1986 & 1987 & 1988 & 1989 & 1990 & 1991 & 1992 & 1993 & 1994 & 1995 & Média \\
\hline Aurora - 1 & $160 \mathrm{a}$ & $163 \mathrm{abcd}$ & $88 \mathrm{~b}$ & $155 \mathrm{abc}$ & $66 \mathrm{bcdef}$ & $94 \mathrm{cde}$ & $100 \mathrm{a}$ & $120 \mathrm{~b}$ & $74 \mathrm{abc}$ & $99 \mathrm{abc}$ & 112 \\
Talismã & $54 \mathrm{cdef}$ & $200 \mathrm{a}$ & $55 \mathrm{de}$ & $147 \mathrm{abc}$ & $125 \mathrm{a}$ & $120 \mathrm{bc}$ & $43 \mathrm{bcdef}$ & $59 \mathrm{def}$ & $75 \mathrm{abc}$ & $112 \mathrm{a}$ & 99 \\
Régis & $98 \mathrm{bc}$ & $91 \mathrm{de}$ & $82 \mathrm{~b}$ & $191 \mathrm{a}$ & $41 \mathrm{ef}$ & $169 \mathrm{a}$ & $43 \mathrm{bcdef}$ & $89 \mathrm{bcd}$ & $85 \mathrm{ab}$ & $95 \mathrm{abc}$ & 98 \\
IAC 680 - 13 & $61 \mathrm{cdef}$ & $145 \mathrm{abcde}$ & $51 \mathrm{def}$ & $115 \mathrm{bc}$ & $53 \mathrm{cdef}$ & $109 \mathrm{bcde}$ & $57 \mathrm{bcd}$ & $169 \mathrm{a}$ & $94 \mathrm{a}$ & $93 \mathrm{abc}$ & 95 \\
IAC 680 - 178 & $147 \mathrm{ab}$ & $139 \mathrm{abcde}$ & $37 \mathrm{f}$ & $162 \mathrm{ab}$ & $53 \mathrm{cdef}$ & $102 \mathrm{bcde}$ & $53 \mathrm{bcde}$ & $85 \mathrm{bcde}$ & $75 \mathrm{abc}$ & $89 \mathrm{abc}$ & 94 \\
Flordaprince & $90 \mathrm{~cd}$ & $196 \mathrm{ab}$ & $40 \mathrm{ef}$ & $85 \mathrm{a}$ & $106 \mathrm{ab}$ & $111 \mathrm{bcd}$ & $41 \mathrm{bcdef}$ & $109 \mathrm{bc}$ & $50 \mathrm{cdef}$ & $82 \mathrm{abc}$ & 91 \\
Centenária & $90 \mathrm{~cd}$ & $119 \mathrm{cdef}$ & $74 \mathrm{~b}$ & $124 \mathrm{abc}$ & $107 \mathrm{ab}$ & $106 \mathrm{bcde}$ & $67 \mathrm{~b}$ & $74 \mathrm{cde}$ & $49 \mathrm{def}$ & $96 \mathrm{abc}$ & 90 \\
Tropical & $104 \mathrm{bc}$ & $125 \mathrm{bcde}$ & $110 \mathrm{a}$ & $163 \mathrm{ab}$ & $52 \mathrm{cdef}$ & $81 \mathrm{def}$ & $35 \mathrm{cdef}$ & $76 \mathrm{cde}$ & $57 \mathrm{cde}$ & $91 \mathrm{abc}$ & 89 \\
Jóia - 1 & $75 \mathrm{cde}$ & $81 \mathrm{e}$ & $77 \mathrm{~b}$ & $111 \mathrm{bc}$ & $85 \mathrm{abcd}$ & $121 \mathrm{bc}$ & $65 \mathrm{bc}$ & $87 \mathrm{bcd}$ & $97 \mathrm{a}$ & $66 \mathrm{c}$ & 89 \\
IAC 2380 - 55 & $89 \mathrm{~cd}$ & $86 \mathrm{e}$ & $91 \mathrm{~b}$ & $97 \mathrm{bc}$ & $81 \mathrm{bcde}$ & $132 \mathrm{ab}$ & $20 \mathrm{f}$ & $28 \mathrm{f}$ & $68 \mathrm{bcd}$ & $108 \mathrm{~b}$ & 80 \\
Josefina & $98 \mathrm{bc}$ & $120 \mathrm{bcde}$ & $84 \mathrm{~b}$ & $130 \mathrm{abc}$ & $26 \mathrm{f}$ & $73 \mathrm{ef}$ & $25 \mathrm{def}$ & $53 \mathrm{def}$ & $40 \mathrm{ef}$ & $71 \mathrm{bc}$ & 72 \\
Rubro - sol & $17 \mathrm{f}$ & $174 \mathrm{abc}$ & $57 \mathrm{cde}$ & $163 \mathrm{ab}$ & $86 \mathrm{abc}$ & $27 \mathrm{~g}$ & $22 \mathrm{ef}$ & $45 \mathrm{ef}$ & $29 \mathrm{fg}$ & $96 \mathrm{abc}$ & 71 \\
Del. precoce & $38 \mathrm{def}$ & $139 \mathrm{abcde}$ & $59 \mathrm{~b}$ & $122 \mathrm{abc}$ & $61 \mathrm{cdef}$ & $50 \mathrm{fg}$ & $19 \mathrm{f}$ & $71 \mathrm{cde}$ & $30 \mathrm{fg}$ & $63 \mathrm{c}$ & 65 \\
IAC 2680 - 91 & $34 \mathrm{ef}$ & $115 \mathrm{cde}$ & $53 \mathrm{def}$ & $150 \mathrm{abc}$ & $42 \mathrm{def}$ & $76 \mathrm{def}$ & $28 \mathrm{def}$ & $28 \mathrm{f}$ & $12 \mathrm{~g}$ & $74 \mathrm{abc}$ & 61 \\
\hline F (Trat.) & $19,29 * *$ & $8,70 * *$ & $7,21 * *$ & $5,73 * *$ & $14,72 * *$ & $3,06 * *$ & $16,87 * *$ & $29,02 * *$ & $36,85^{* *}$ & $3,52 * *$ & -- \\
CV (\%) & 24,82 & 21,11 & 28,77 & 20,22 & 4,74 & 14,56 & 27,29 & 20,56 & 15,85 & 18,07 & --- \\
\hline
\end{tabular}

${ }^{1}$ Valores nas colunas, seguidos da mesma letra, não diferem entre si a $5 \%$ de probabilidade, pelo teste Tukey

** Significativo a $1 \%$

TABELA2. Produção média de frutos (kg) por planta, de cultivares e seleções de pessegueiros e nectarineiras no espaçamento 4 x 1,5 m (1.667 pl/ha), durante o período de 1986 a 1995 . Estação Experimental de Monte Alegre do Sul, IAC'1.

\begin{tabular}{|c|c|c|c|c|c|c|c|c|c|c|c|}
\hline Material & 1986 & 1987 & 1988 & 1989 & 1990 & 1991 & 1992 & 1993 & 1994 & 1995 & Média \\
\hline Aurora - 1 & $11,17 \mathrm{a}$ & $11,85 \mathrm{abc}$ & $7,51 \mathrm{ab}$ & $12,03 a b c$ & 6,07abcde & $9,30 \mathrm{bc}$ & $8,32 \mathrm{a}$ & $10,97 \mathrm{ab}$ & $6,46 a b c$ & $8,10 \mathrm{abc}$ & 9,2 \\
\hline IAC $680-178$ & $10,52 \mathrm{ab}$ & $11,48 \mathrm{abc}$ & $3,75 b$ & $15,43 \mathrm{a}$ & $4,55 \mathrm{cdef}$ & $10,43 a b$ & $4,59 b c$ & $9,44 \mathrm{bcd}$ & $6,76 \mathrm{ab}$ & $8,41 \mathrm{abc}$ & 8,5 \\
\hline IAC $680-13$ & $5,75 \mathrm{~cd}$ & $12,41 \mathrm{ab}$ & $4,73 \mathrm{ab}$ & $10,84 \mathrm{abc}$ & 3,89def & 8,91 bcd & 4,09bcd & $14,39 a$ & $7,61 \mathrm{a}$ & $8,23 \mathrm{abc}$ & 8,1 \\
\hline Talismã & $3,93 \mathrm{cde}$ & $11,76 a b c$ & $5,80 \mathrm{ab}$ & $12,96 a b c$ & $9,40 \mathrm{a}$ & $11,40 \mathrm{ab}$ & $5,20 \mathrm{~b}$ & $4,80 \mathrm{def}$ & $5,67 \mathrm{abcd}$ & $10,30 \mathrm{a}$ & 8,1 \\
\hline Régis & $6,16 \mathrm{c}$ & 7,90bcde & $6,10 \mathrm{ab}$ & $14,48 \mathrm{ab}$ & $3,41 \mathrm{def}$ & $13,32 \mathrm{a}$ & $3,73 \mathrm{bcd}$ & $8,81 \mathrm{bcd}$ & $6,25 \mathrm{abc}$ & $8,33 \mathrm{abc}$ & 7,8 \\
\hline Flordaprince & $7,09 \mathrm{bc}$ & $13,85 \mathrm{a}$ & $4,21 \mathrm{ab}$ & $8,43 b c$ & $8,63 a b$ & $10,70 \mathrm{ab}$ & $3,02 \mathrm{bcd}$ & $10,74 a b c$ & 4,00de & $6,62 \mathrm{abc}$ & 7,7 \\
\hline Jóia - 1 & $6,16 \mathrm{c}$ & $6,67 \mathrm{de}$ & $6,37 \mathrm{ab}$ & $8,64 b c$ & $6,70 \mathrm{abcd}$ & $9,00 \mathrm{bcd}$ & $5,07 \mathrm{~b}$ & 7,75 bcde & $7,32 \mathrm{a}$ & $4,68 \mathrm{abc}$ & 6,8 \\
\hline Centenária & $6,12 \mathrm{c}$ & 7,38cde & $6,19 a b$ & $12,03 \mathrm{abc}$ & $8,04 \mathrm{abc}$ & $7,66 \mathrm{bcd}$ & $5,13 b$ & $5,12 \mathrm{def}$ & $2,40 \mathrm{ef}$ & $6,71 \mathrm{abc}$ & 6,7 \\
\hline Tropical & $6,04 \mathrm{c}$ & 7,90bcde & $8,43 \mathrm{a}$ & $12,16 \mathrm{abc}$ & 4,32def & $6,11 \mathrm{~cd}$ & $2,81 \mathrm{bcd}$ & $6,11 \mathrm{cdef}$ & $4,56 \mathrm{bcde}$ & 7,03abc & 6,5 \\
\hline Del. precoce & $3,90 \mathrm{cde}$ & $11,48 \mathrm{abc}$ & $6,48 \mathrm{ab}$ & $10,27 \mathrm{abc}$ & 5,59abcde & $6,38 \mathrm{~cd}$ & $1,68 \mathrm{~d}$ & 6,68 bcdef & $2,70 \mathrm{ef}$ & $6,61 \mathrm{abc}$ & 6,2 \\
\hline IAC $2380-55$ & $5,47 \mathrm{~cd}$ & $5,55 \mathrm{e}$ & 7,20ab & $7,55 \mathrm{c}$ & 5,57 bcde & $8,49 \mathrm{bcd}$ & $1,45 \mathrm{~d}$ & $1,99 \mathrm{f}$ & 4,45 cde & $8,47 a b$ & 5,6 \\
\hline Josefina & $5,40 \mathrm{~cd}$ & 8,02 bcde & $6,06 a b$ & $9,02 \mathrm{abc}$ & $2,04 \mathrm{f}$ & 5,20 de & $1,62 d$ & $4,07 \mathrm{ef}$ & $2,82 \mathrm{ef}$ & $4,81 \mathrm{bc}$ & 4,9 \\
\hline Rubro - sol & $0,88 \mathrm{e}$ & 10,19abcd & $4,06 \mathrm{~b}$ & $10,64 \mathrm{abc}$ & $4,15 \mathrm{def}$ & $1,48 \mathrm{e}$ & $1,60 \mathrm{~d}$ & $2,94 \mathrm{f}$ & $1,40 \mathrm{f}$ & $7,18 \mathrm{abc}$ & 4,4 \\
\hline IAC $2680-91$ & 2,09de & $7,26 \mathrm{cde}$ & $4,05 \mathrm{~b}$ & $9,14 \mathrm{abc}$ & $2,90 \mathrm{ef}$ & $5,20 \mathrm{de}$ & $2,23 \mathrm{~cd}$ & $2,16 \mathrm{f}$ & $0,90 \mathrm{f}$ & $4,66 \mathrm{c}$ & 4,0 \\
\hline at.) & $18,42 * *$ & $10,63 * *$ & $4,18 * *$ & $4,10 * *$ & $13,40 * *$ & $21,26 * *$ & $16,64 * *$ & $20,59 * *$ & $37,40 * *$ & $8,81 * *$ & --- \\
\hline CV (\%) & 24,88 & 18,61 & 27,83 & 23,59 & 24,91 & 18,24 & 29,53 & 26,38 & 17,92 & 9,24 & --- \\
\hline
\end{tabular}

${ }^{1}$ Valores nas colunas, seguidos da mesma letra, não diferem entre si a 5\% de probabilidade, pelo teste Tukey.

** Significativo a $1 \%$ 
Em alguns anos, as cultivares Aurora-1, IAC 680-178, IAC 680-13, Talismã, Flordaprince, Centenária e Tropical ultrapassaram a $20 \mathrm{t} / \mathrm{ha}$, índices esses comparáveis com os melhores resultados obtidos na Europa em espaçamentos de 4 x 1,5 m. Os piores desempenhos foram verificados nas nectarineiras Rubro-sol, IAC N 2680-91 e Josefina, além do pessegueiro IAC 2380-55. Estas cultivares e seleções não apresentaram, em geral, recuperação regular da copa ao longo dos anos.

Na Tabela 3, são apresentadas várias características das diversas cultivares e seleções, determinadas no décimo ano de produção. Não foram observadas diferenças significativas no volume de copa entre os pessegueiros e nectarineiras. Ao contrário, ao longo dos anos houve, entre esses pessegueiros e nectarineiras um crescimento diferencial, medido pela área da secção do tronco. Essa diferença no crescimento, que atingiu até $130 \%$, pode ser atribuída principalmente às características genéticas das cultiva- res em pesquisa. O crescimento diferencial não alterou significativamente o desenvolvimento da copa, o que foi confirmado pela baixa correlação $(\mathrm{r}=0,45)$ entre a área da secção transversal do tronco e o volume da copa (Tabela 4). A baixa correlação pode ter sido conseqüência do crescimento contínuo do tronco ao longo dos anos, ao passo que o volume da copa foi ajustado a cada dois anos pelo sistema de poda utilizado. Além disso, nas maiores densidades populacionais ocorre menor desenvolvimento da copa, em conseqüência do alto nível de competição por luz, água, nutrientes e espaço físico (Bargioni et al., 1985; Recupero et al., 1985; Barbosa et al., 1989).

No pessegueiro, nem sempre tem sido obtida uma alta correlação entre a área da secção transversal do tronco e o tamanho da copa, mesmo entre plantas de uma mesma cultivar (Chalmers \& Van Den Ende, 1975). Ao contrário do volume da copa, todas as demais características fenotípicas apresentaram diferenças

TABELA3. Desenvolvimento e produção de frutos de cultivares e seleções de pessegueiros e nectarineiras em pomar compacto ( 4 x 1,5 m), no décimo ano de experimentação, na Estação Experimental de Monte Alegre do Sul, IAC. Valores médios obtidos em cinco plantas por cultivar e seleção no ano agrícola de 1995 .

\begin{tabular}{|c|c|c|c|c|c|c|c|c|}
\hline \multirow[t]{3}{*}{ Material } & \multicolumn{8}{|c|}{ Características fisiológicas } \\
\hline & \multirow{2}{*}{$\begin{array}{c}\text { Área } \\
\text { transversal } \\
\text { do tronco }\end{array}$} & \multirow{2}{*}{$\begin{array}{l}\text { Volume } \\
\text { da copa }\end{array}$} & \multirow{2}{*}{$\begin{array}{c}\text { Área } \\
\text { da folha }\end{array}$} & \multicolumn{3}{|c|}{ Produção de frutos por planta } & \multicolumn{2}{|c|}{ Índices fisiológicos } \\
\hline & & & & Fruto & Massa & $\begin{array}{l}\text { Massa } \\
\text { média }\end{array}$ & $\begin{array}{c}\text { Frutos } / \mathrm{cm}^{2} \\
\text { de tronco }\end{array}$ & $\begin{array}{r}\text { Frutos } / \mathrm{cm}^{2} \\
\text { de tronco }\end{array}$ \\
\hline & $\left(\mathrm{cm}^{2}\right)$ & $\left(\mathrm{m}^{3}\right)$ & $\left(\mathrm{cm}^{2}\right)$ & $\left(\mathrm{n}^{\mathrm{o}}\right)$ & $(\mathrm{kg})$ & $(\mathrm{g})$ & $\left(\mathrm{n}^{\circ} / \mathrm{cm}^{2}\right)$ & $\left(\mathrm{g} / \mathrm{cm}^{2}\right)$ \\
\hline Aurora - 1 & $146,3 \mathrm{~cd}$ & 8,60 & $33,93 \mathrm{abc}$ & $99 \mathrm{abc}$ & $8,10 \mathrm{abc}$ & $81,8 \mathrm{bcd}$ & $0,68 \mathrm{ab}$ & $55,4 \mathrm{ab}$ \\
\hline IAC $680-178$ & $162,0 \mathrm{bcd}$ & 7,87 & $40,90 \mathrm{a}$ & $89 \mathrm{abc}$ & $8,41 \mathrm{abc}$ & $94,5 \mathrm{ab}$ & $0,55 \mathrm{ab}$ & $51,9 \mathrm{ab}$ \\
\hline IAC $680-13$ & $278,6 \mathrm{a}$ & 8,30 & $37,46 \mathrm{ab}$ & $93 \mathrm{abc}$ & $8,23 \mathrm{abc}$ & 88,5 abcd & $0,33 b$ & $29,5 b$ \\
\hline Régis & $157,9 \mathrm{bcd}$ & 7,57 & $37,26 \mathrm{abc}$ & $95 \mathrm{abc}$ & $8,33 \mathrm{abc}$ & $87,7 \mathrm{abcd}$ & $0,60 \mathrm{ab}$ & $52,7 \mathrm{ab}$ \\
\hline Talismã & $150,8 \mathrm{bcd}$ & 9,35 & $33,88 \mathrm{abc}$ & $112 \mathrm{a}$ & $10,30 \mathrm{a}$ & $92,0 \mathrm{abc}$ & $0,74 \mathrm{a}$ & $68,3 \mathrm{a}$ \\
\hline Flordaprince & $214,9 \mathrm{abc}$ & 8,15 & $28,44 \mathrm{c}$ & $82 \mathrm{abc}$ & $6,62 \mathrm{abc}$ & $80,7 \mathrm{bcd}$ & $0,38 \mathrm{ab}$ & $30,8 b$ \\
\hline Jóia - 1 & $130,8 \mathrm{~d}$ & 7,70 & $29,87 b c$ & $66 c$ & $4,68 b c$ & $70,9 \mathrm{de}$ & $0,50 \mathrm{ab}$ & $35,7 \mathrm{ab}$ \\
\hline Centenária & $204,2 \mathrm{abcd}$ & 9,22 & $35,25 \mathrm{abc}$ & $96 a b c$ & $6,71 \mathrm{abc}$ & $69,8 \mathrm{de}$ & $0,47 a b$ & $32,8 b$ \\
\hline Tropical & $237,4 \mathrm{ab}$ & 8,47 & $33,03 \mathrm{abc}$ & $91 \mathrm{abc}$ & 7,03abc & $77,2 \mathrm{bcd}$ & $0,38 \mathrm{ab}$ & $29,6 b$ \\
\hline Del. precoce & $119,7 d$ & 6,25 & $33,15 \mathrm{abc}$ & $63 c$ & $6,61 \mathrm{abc}$ & $104,9 \mathrm{a}$ & $0,53 \mathrm{ab}$ & $55,2 \mathrm{ab}$ \\
\hline IAC $2380-55$ & $159,5 \mathrm{bcd}$ & 8,62 & $38,44 \mathrm{ab}$ & $108 \mathrm{ab}$ & $8,47 \mathrm{ab}$ & $78,4 \mathrm{bcd}$ & $0,67 \mathrm{ab}$ & $53,1 \mathrm{ab}$ \\
\hline Josefina & $213,7 \mathrm{abc}$ & 8,70 & $33,12 \mathrm{abc}$ & $71 b c$ & $4,81 \mathrm{bc}$ & $67,7 \mathrm{~cd}$ & $0,33 b$ & $22,5 b$ \\
\hline IAC $2680-91$ & $126,4 \mathrm{~cd}$ & 6,62 & $32,12 \mathrm{abc}$ & $74 a b c$ & $4,66 \mathrm{c}$ & $62,9 \mathrm{cde}$ & $0,58 \mathrm{ab}$ & $36,8 \mathrm{ab}$ \\
\hline Rubro - sol & $190,4 \mathrm{abcd}$ & 7,75 & $33,75 \mathrm{abc}$ & $96 a b c$ & $7,18 \mathrm{abc}$ & $74,7 \mathrm{cde}$ & $0,50 \mathrm{ab}$ & $37,7 \mathrm{ab}$ \\
\hline F (Trat.) & $7,10 * *$ & ns & $4,57 * *$ & $3,52 * *$ & $4,81 * *$ & $8,81 * *$ & $3,50 * *$ & 4,28 \\
\hline $\mathrm{CV}(\%)$ & 19,86 & 22,33 & 10,14 & 18,03 & 21,06 & 9,24 & 27,44 & 28,98 \\
\hline
\end{tabular}

${ }^{1}$ Valores nas colunas, seguidos da mesma letra, não diferem entre si a 5\% de probabilidade, pelo teste Tukey.

** Significativo a $1 \%$ 
TABELA 4. Coeficientes de correlação simples entre diversas características fisiológicas da produção em cultivares e seleções de pessegueiros e nectarineiras cultivados em pomar compacto, sob poda drástica bienal. Instituto Agronômico (IAC), 1995.

\begin{tabular}{lc}
\hline Correlações & Coeficientes de correlação simples \\
\hline Área da secção do tronco x volume da copa & $0,45^{\mathrm{ns}}$ \\
Área da secção do tronco x número de frutos & $0,18^{\mathrm{ns}}$ \\
Área da secção do tronco x massa de frutos & $0,07^{\mathrm{ns}}$ \\
Volume da copa x número de frutos & $0,67^{* *}$ \\
Massa total de frutos x massa média de frutos & $0,51^{*}$ \\
\hline
\end{tabular}

* Significativo a $5 \%$.

** Significativo a $1 \%$

ns Não-significativo.

significativas, cuja causa principal pode ser atribuída às características genotípicas dos híbridos (Tabela 3).

Neste trabalho, a intensidade de desbaste dos frutos foi baseada no número de ramos, ou seja, em um dos principais componentes do volume da copa. Os dados mostram ser este um critério adequado, pois foi obtida uma alta correlação entre o volume da copa e a produção em número de frutos. O contrário ocorreu com a correlação entre a área da secção transversal do tronco e o número de frutos, que apresentou valor bastante baixo (Tabela 4).

O cultivo adensado de pessegueiro e nectarineira, associado à poda drástica bienal, leva à obtenção de baixas relações de massa $\left(22,5\right.$ a $\left.68,3 \mathrm{~g} / \mathrm{cm}^{2}\right)$ e do número de frutos $\left(0,33\right.$ a 0,74 frutos $\left./ \mathrm{cm}^{2}\right)$ por secção transversal do tronco (Tabela 3 ). Comparando-se os dados obtidos em plantas manejadas tradicionalmente, verificam-se valores de $94 \mathrm{~g} / \mathrm{cm}^{2}$ (Scorza et al., 1986) e cerca de 5 frutos $/ \mathrm{cm}^{2}$ de àrea da secção transversal de tronco (Feliciano et al., 1984). A relação de cerca de 5 frutos por $\mathrm{cm}^{2}$ de área da secção transversal do tronco, em sistema compacto, levaria a um número excessivamente alto de pêssegos $\mathrm{e}$ nectarinas por planta. Com o aumento da idade da planta, do crescimento contínuo do tronco e da manutenção aproximadamente constante do volume da copa, a citada relação causaria diminuição drástica no tamanho dos frutos. A correlação obtida entre a massa total e média dos frutos $(\mathrm{r}=0,51)$ mostra que 0 índice adotado para o desbaste foi adequado. Portanto, o desbaste de frutos baseado no número de ramos, ou seja, no volume da copa, parece ser o índice mais indicado para o sistema adensado de plantio de pessegueiros e nectarineiras submetidos à poda drástica bienal.

Ressalte-se, ainda, que as plantas, após os dez anos de cultivo, mantinham uma produção normal, semelhante à média decenal. Isso permite prever uma vida média bem longa para as cultivares e seleções mais rústicas, característica extremamente desejável ao sistema de pomar compacto.

\section{CONCLUSÕES}

1. As cultivares e seleções Aurora-1, IAC 680-178, IAC 680-13, Régis, Talismã e Flordaprince revelam-se como as mais produtivas no sistema pesquisado.

2. As cultivares e seleções de pêssegos e nectarinas utilizadas no experimento demonstram, no sistema de pomar compacto com poda drástica da copa, grande potencial de longevidade das plantas.

\section{AGRADECIMENTOS}

Ao pessoal de apoio, Lázaro de Godoi, Meire Correia da Silva Ferrari, Marcilene de Moraes e Antonio Carlos de Carvalho, pelos tratos culturais das plantas e pelo levantamento de dados dos experimentos; e à pesquisadora científica, Maria do Carmo de Salvo Soares Novo, pela colaboração na análise estatística dos caracteres fisiológicos. 


\section{REFERÊNCIAS}

BARBOSA, W. Desenvolvimento vegetativo e reprodutivo do pessegueiro em pomar compacto sob poda drástica anual. Piracicaba: ESALQ, 1989. 154p. Dissertação de Mestrado.

BARBOSA, W.; CAMPO DALL'ORTO, F.A.; OJIMA, $M$. Comportamento vegetativo e reprodutivo do pêssego Tropical: cultivar bem precoce no Planalto Paulista. Campinas: IAC, 1990a. 22p. (Boletim Científico, 21).

BARBOSA, W.; CAMPO DALL'ORTO, F.A.; OJIMA, M. O pessegueiro no sistema de pomar compacto: I. Conjecturas, experimentação e prática. O Agronômico, Campinas, v.41, n.1, p.26-39, 1989.

BARBOSA, W.; CAMPO DALL'ORTO, F.A.; OJIMA, $\mathrm{M}$. O pessegueiro no sistema de pomar compacto: V. Pesquisas do Instituto Agronômico na década de 80. O Agronômico, Campinas, v.42, n.1, p.35-44, $1990 \mathrm{~b}$

BARBOSA, W.; CAMPO DALL'ORTO, F.A.; OJIMA, M.; LOVATE, A.A.; SANTOS, R.R. O pessegueiro no sistema de pomar compacto: VII. Comportamento de novas seleções IAC sob poda drástica bienal. Scientia Agricola, v.51, n.1, p.94-98, 1994.

BARBOSA, W.; CAMPO DALL'ORTO, F.A.; OJIMA, M.; MARTINS, F.P.; IGUE, T. O pessegueiro no sistema de pomar compacto: IV. Intensidade e época de raleio dos frutos das cultivares Tropical e Aurora-1. Bragantia, Campinas, v.50, n.1, p.91-102, 1991a.

BARBOSA, W.; CAMPO DALL'ORTO, F.A.; OJIMA, M.; SAMPAIO, V.R. O pessegueiro no sistema de pomar compacto: III. Épocas de poda drástica na diferenciação floral. Bragantia, Campinas, v.49, n.1, p. 147-155, 1990c.

BARBOSA, W.; CAMPO DALL'ORTO, F.A.; OJIMA, M.; SANTOS, R.R. O pessegueiro no sistema de pomar compacto: VI. Frutificação efetiva e raleio químico em seleções IAC. Bragantia, Campinas, v.51, n.1, p.63-67, 1992

BARBOSA, W.; CAMPO DALL'ORTO, F.A.; OJIMA, M.; SANTOS, R.R. O pessegueiro no sistema de pomar compacto: VII - Caracterização de cultivares e seleções em três densidades populacionais. Campinas: Instituto Agronômico, 1996. 12p. (Documentos IAC, 53).
BARBOSA, W.; OJIMA, M.; CAMPO DALL'ORTO, F.A.; MARTINS, F.P. Época e ciclo de maturação de pêssegos e nectarinas no Estado de São Paulo. Bragantia, Campinas, v.49, n.2, p.221-226, 1990d.

BARBOSA, W.; OJIMA, M.; CAMPO DALL'ORTO, F.A.; RIGITANO, O.; MARTINS, F.P.; SANTOS, R.R.; CASTRO, J.L. Melhoramento do pessegueiro para regiões de clima subtropical-temperado: realizações do Instituto Agronômico no período de 1950/1990. Campinas: IAC, 1997. 22p. (Documentos IAC, 52).

BARBOSA, W.; OJIMA, M.; CAMPO DALL'ORTO, F.A.; SAMPAIO, V.R.; MARTINS, F.P.; IGUE, T. $O$ pessegueiro no sistema de pomar compacto: II. Influência das épocas de poda drástica no desenvolvimento das plantas. Campinas: IAC, 1991b. 20p. (Boletim Científico, 22).

BARGIONI, G.; LORETI, F.; PISANI, P.L. Osservazioni sulla coltivazione del pesco ad elevate densità di piantagione. Rivista della Ortoflorofrutticoltura Italiana, Firenze, v.2, p.147-159, 1979

BARGIONI, G.; LORETI, F.; PISANI, P.L. Ten years of research on peach and nectarine in a hight density system in the Verona area. Acta Horticulturae, Wageningen, v.173, p.229-309, 1985

CAMPO DALL'ORTO, F.A.; OJIMA, M.; BARBOSA, W.; MARTINS, F.P. O nanismo do pessegueiro induzido pela enxertia no Damasqueiro-Japonês. Pesquisa Agropecuária Brasileira, Brasília, v.27. n.3, p.517-521, mar. 1992.

CAMPO DALL'ORTO, F.A.; OJIMA, M.; BARBOSA, W.; MARTINS, F.P.; RIGITANO, O. 'Delicioso Precoce' - nova seleção de pêssego do IAC para as regiões mais frias de São Paulo. Pesquisa Agropecuária Brasileira, Brasília, v.24, n.2, p.233-239 , fev. 1989

CAMPO DALL'ORTO, F.A.; OJIMA, M.; BARBOSA, W.; TOMBOLATO, A.F.C.; RIGITANO, O.; ALVES, S. Cultivo de seleções de pessegueiros precoces no sistema de pomar compacto com poda drástica anual de renovação da copa. Pesquisa Agropecuária Brasileira, Brasília, v.19, n.6, p.719-727, jun. 1984.

CHALMERS, D.J.; Van Den ENDE, B. Productivity of peach trees: factors affecting dry-weight distribution during tree growth. Annals of Botany, London, v.39, p.423-432, 1975. 
EREZ, A. Adaptation of the peach to the meadow orchard system. Acta Horticulturae, Wageningen, v.65, p.245-250, 1978.

EREZ, A. Peach meadow orchards. Acta Horticulturae, Wageningen, v.173, p.405-411, 1985.

FELICIANO, A.J.; NAKASU, B.H.; SACHS, S Raleio. In: EMBRAPA. Centro Nacional de Pesquisa de Fruteiras de Clima Temperado (Pelotas, RS). A cultura do pessegueiro. Pelotas, 1984. 156p. (Embrapa-CNPFCT. Circular técnica, 10).

GUERRIERO, B.; LORETI, F.; NATALI, S. Eight years of observations on a peach double-row planted orchard. Acta Horticulturae, v.114, p.362-363, 1980 .

LORETI, E.; MASSAI, B; MORINI, S. Effect of training system, planting density and rootstock on growth and quality of peach shoots. Advances in Horticultural Science, New York, v.3, p.45-47, 1991.

OJIMA, M.; CAMPO DALL'ORTO, F.A.; BARBOSA, W.; RIGITANO, O. Frutas de Clima Temperado. In: FURLANI, A.M.C.; VIÉGAS, G.P. (Eds.). O Melhoramento de Plantas no Instituto Agronômico. Campinas: Instituto Agronômico, 1993. 524p.

OJIMA, M.; CAMPO DALL'ORTO, F.A.; BARBOSA, W.; RIGITANO, O. Desenvolvimento da fruticultura de clima temperado em São Paulo: contri- buição do Instituto Agronômico até seu Centenário 1887/1987. Campinas: IAC, 1988. 63p. (Documentos IAC, 11).

PEDRO JUNIOR, M.J.; ORTOLANI, A.A.; RIGITANO, O.; ALFONSI, R.R.; PINTO, H.S.; BRUNINI, O. Estimativa de horas de frio abaixo de 7 e $13^{\circ} \mathrm{C}$ para regionalização da fruticultura de clima temperado no Estado de São Paulo. Bragantia, Campinas, v.38, p.123-130, 1979.

RECUPERO, S.; MONASTRA, F.; DAMIANO, C. Study on planting densities of peach and nectarine cultivars on different rootstocks. Acta Horticulturae, Wageningen, v.173, n.3, p.311-322, 1985.

REIS, G.G; MÜLLER, M.W. Análise de crescimento de plantas: mensuração do crescimento. Belém: Universidade de Ciências Agrárias do Pará. Serviço de Documentação e Informação, 1979. 39p. (Informe Didático, 1).

SCORZA, R.; ZAILONG, L.; LIGHTNER, G.W.; GILREATH, L.E. Dry matter distribution and responses to pruning within a population of standard, semidwarf compact, and dwarf peach seedlings. Journal of the American Society for Horticultural Science, v.111, n.4, p.541-545, 1986.

WESTWOOD, M.N. Temperate zone pomology. San Francisco: W.H.Freeman, 1978. 428p. 